\title{
COMPARISONS BETWEEN A DENSE MEDIUM SCATTERING MODEL AND SEAICE MEASUREMENTS
}

\author{
Saibun Tjuatja, Adrian K. Fung, and Jonathan Bredow \\ Wave Scattering Research Center \\ University of Texas at Arlington, UTA Box 19016 \\ Arlington, Texas 76019, U.S.A.
}

\section{ABSTRACT}

A scattering model for a plane dense medium layer embeded with randomly located small spherical scatterers was developed. The effects of close spacing between the scatterers were included in the developed phase matrix by keeping the range-dependent terms in the scattered fields expressions. The derived phase matrix was then used in the radiative transfer formulation to model the scattering from a dense medium layer. The model was used in the calculations of backscattering coefficients of multi year Arctic sea ice.

Key words: volume scatter, dense medium, sea ice

\section{INTRODUCTION}

In a dense and nontenuous medium, the scatterers occupy an appreciable fractional volume, and their dielectric property differ significantly from that of the background medium. Previous studies on the propagation and scattering of wave in dense nontenuous media have assumed either the scatterers are in the far field of one another [1,2] or the scatterers are of point size. The far field assumption is fairly good only when the scatterers are small and the average spacing between scatterers is larger than $\lambda / 3$, where $\lambda$ is the incident wavelength [3]. However, in some naturally occuring dense media, such as snow and multi year sea ice, neither the far-field nor the point size scatterers assumptions are valid. In order to account for some of the near field and scatterer size effects, additional range and size dependent terms in the scattered fields are included in the calculation of the phase matrix. It is demonstrated that the inclusion of these terms affects the backscattering.

The development of the phase matrix is described in the next section. This phase matrix is then used in a scattering model to estimate the parameters of a multi year thick sea ice layer and compare with sea ice measurement results $[4,5]$.

\section{THEORETICAL FORMULATION}

Consider a time harmonic plane wave incident on a dielectric sphere (Fig. 1.). The sphere has radius "a" , relative permittivity $\varepsilon_{\mathrm{T}}\left(\varepsilon_{\mathrm{T}}=\varepsilon_{\mathrm{T}}{ }^{\prime}+j \varepsilon_{\mathrm{r}}{ }^{\prime \prime}\right)$, and permeability $\mu$ equal to that of the background medium. The incident electric and magnetic field components (with $\mathrm{e}^{-\mathrm{j} \omega t}$ suppressed) are

$$
\begin{aligned}
& \overline{E_{i}}=\widehat{x} E_{0} e^{j k z} \\
& \overline{H_{i}}=\widehat{y} \frac{E_{0}}{\eta} e^{j k z}
\end{aligned}
$$

where

$$
\begin{aligned}
& k=\omega \sqrt{\mu \varepsilon} \\
& \eta=\sqrt{\frac{\mu}{\varepsilon}}
\end{aligned}
$$

and $\varepsilon$ is the permittivity of the background medium. The scattered $\mathrm{E}$ and $\mathrm{H}$ fields due to the sphere are given by $[6,7]$

$$
\begin{aligned}
\overline{E^{s}}=E_{0} \sum_{n=1}^{\infty}\left[j^{n} \frac{2 n+1}{n(n+1)}\right] \\
\cdot\left[a_{n} \bar{m}_{o 1 n}^{(3)}-j b_{n} \bar{n}_{e 1 n}^{(3)}\right] \\
\overline{H^{s}}=\frac{E_{0}}{\eta} \sum_{n=1}^{\infty}\left[j \frac{2 n+1}{n(n+1)}\right] \\
\cdot\left[b_{n} \bar{m}_{e l n}^{(3)}-j a_{n} \bar{n}_{o 1 n}^{(3)}\right]
\end{aligned}
$$

where

$$
\begin{aligned}
\overline{\mathrm{m}}_{\mathrm{e}}^{(3)}{ }_{1 \mathrm{n}}= & \pm \frac{\hat{\theta}}{\sin \theta} \mathrm{h}_{\mathrm{n}}^{(1)}(\mathrm{kr}) \mathrm{P}_{\mathrm{n}}^{1}(\cos \theta) \begin{array}{r}
\sin \phi \\
\cos \phi
\end{array} \\
& -\hat{\phi} \mathrm{h}_{\mathrm{n}}^{(1)}(\mathrm{kr}) \frac{\partial\left\{\mathrm{P}_{\mathrm{n}}^{1}(\cos \theta)\right\}}{\partial \theta} \sin \phi \\
\partial \theta & \cos \phi
\end{aligned}
$$




$$
\begin{aligned}
\hat{n}_{0}^{(3)}{ }_{1 \mathrm{n}} & =\hat{\mathrm{r}} \frac{\mathrm{n}(\mathrm{n}+1)}{\mathrm{kr}} \mathrm{h}_{\mathrm{n}}^{(1)}(\mathrm{kr}) \mathrm{P}_{\mathrm{n}}^{1}(\cos \theta) \begin{array}{l}
\sin \phi \\
\cos \phi
\end{array} \\
& +\frac{\hat{\theta}}{\mathrm{kr}}\left[\mathrm{kr} \mathrm{h}_{\mathrm{n}}^{(1)}(\mathrm{kr})\right]^{\prime} \frac{\partial}{\partial \theta}\left\{\mathrm{P}_{\mathrm{n}}^{1}(\cos \theta)\right\} \begin{array}{l}
\sin \phi \\
\cos \phi
\end{array} \\
& \pm \frac{\hat{\phi}}{\mathrm{kr} \sin \theta}\left[\mathrm{kr} h_{\mathrm{n}}^{(1)}(\mathrm{kr})\right]^{\prime} \mathrm{P}_{\mathrm{n}}^{1}(\cos \theta) \begin{array}{l}
\cos \phi \\
\sin \phi
\end{array}
\end{aligned}
$$

In $(3,4) a_{n}$ and $b_{n}$ are the scattered field Mie coefficients.

$$
\begin{aligned}
& a_{n}=\frac{\sqrt{\varepsilon_{\mathrm{T}}} \hat{\mathrm{J}}_{\mathrm{n}}^{\prime}(\mathrm{x}) \hat{\mathrm{J}}_{\mathrm{n}}(\mathrm{y})-\hat{\mathrm{J}}_{\mathrm{n}}(\mathrm{x}) \hat{\mathrm{J}}_{\mathrm{n}}^{\prime}(\mathrm{y})}{\sqrt{\varepsilon_{\mathrm{r}}}\left\{\hat{\mathrm{H}}_{\mathrm{n}}^{(1)}(\mathrm{x})\right\} \hat{\mathrm{J}}_{\mathrm{n}}(\mathrm{y})-\hat{\mathrm{H}}_{\mathrm{n}}^{(1)}(\mathrm{x}) \hat{\mathrm{J}}_{\mathrm{n}}^{\prime}(\mathrm{y})} \\
& \mathrm{b}_{\mathrm{n}}=\frac{\sqrt{\varepsilon_{\mathrm{r}}} \hat{\mathrm{J}}_{\mathrm{n}}(\mathrm{x}) \hat{\mathrm{J}}_{\mathrm{n}}^{\prime}(\mathrm{y})-\hat{\mathrm{J}}_{\mathrm{n}}^{\prime}(\mathrm{x}) \hat{\mathrm{J}}_{\mathrm{n}}(\mathrm{y})}{\sqrt{\varepsilon_{\mathrm{r}}} \hat{\mathrm{H}}_{\mathrm{n}}^{(1)}(\mathrm{x}) \hat{\mathrm{J}}_{\mathrm{n}}^{\prime}(\mathrm{y})-\left\{\hat{\mathrm{H}}_{\mathrm{n}}^{(1)}\right\}(\mathrm{x}) \hat{\mathrm{J}}_{\mathrm{n}}(\mathrm{y})}
\end{aligned}
$$

where

$$
\begin{aligned}
& x=k \cdot a \\
& y=k_{s} \cdot a=\sqrt{\varepsilon_{r}} k \cdot a
\end{aligned}
$$

In the small sphere scatterer limit $y \rightarrow 0$, (7) and (8) become ill conditioned. The Mie coefficients $a_{1}, a_{2}, b_{1}$, and $b_{2}$ are computed using the methods suggested by Wiscombe [8]. Note that the small scatterer Mie coefficients equations given in Stratton[8] and Ruck[9] yield incorrect results. In $(5,6)$ $P_{n}^{1}(\cos \theta)$ is the associated Legendre Polynomial, $h_{n}^{(1)}(k r)$ is the spherical Hankel function of the first kind. The subscripts o and e denote odd and even. In $(7,8) \hat{\mathrm{J}}_{n}(\mathrm{x})$ and $\widehat{\mathrm{H}}_{\mathrm{n}}^{(1)}(\mathrm{x})$ are the Ricatti-Bessel function and Ricatti-Hankel function of the first kind, respectively; $\varepsilon_{\mathrm{T}}$ is the relative permittivity of the sphere. [ ]' denotes differentiation with respect to the argument.

In low-frequency region $\left(\mathrm{k}_{\mathrm{s}} \mathrm{a}<0.8\right)$, only the first two terms $(n=1,2)$ in (3) and (4) are necessary. The expressions for $\overline{\mathrm{m}}_{\mathrm{e}}^{(3)}{ }_{11}, \overline{\mathrm{m}}_{\mathrm{e}}^{(3)}{ }_{12}, \overrightarrow{\mathrm{n}}_{\mathrm{e}}^{(3)}{ }_{11},{ }_{\mathrm{e}}$, and $\overrightarrow{\mathrm{n}}_{\mathrm{o}}^{(3)}{ }_{12}$ are as follows:

$$
\begin{aligned}
& \overline{\mathrm{m}}_{\mathrm{e}}^{(3)}{ }_{11}=\mathrm{K}_{0} \alpha \frac{\overline{\mathrm{A}}}{\overline{\mathrm{B}}} \\
& \overline{\mathrm{m}}_{\mathrm{e}}^{(3)}{ }_{12}=\mathrm{K}_{0} \beta \frac{\overline{\mathrm{C}_{1}}}{\overline{\mathrm{C}}} \\
& \overline{\mathrm{n}}_{\mathrm{e}}^{(3)}{ }_{11}=\mathrm{K}_{0} \frac{\overline{\mathrm{E}_{1}}}{\overline{\mathrm{E}_{2}}}
\end{aligned}
$$

$$
{ }_{\mathrm{e}}^{-(3)}{ }_{12}=\mathrm{K}_{0} \frac{\overline{F_{1}}}{\bar{F}}
$$

where

$$
\mathrm{K}_{0}=\frac{\mathrm{e}^{\mathrm{j} \mathrm{k}_{\mathrm{s}} \mathrm{r}}}{\mathrm{k}_{\mathrm{s}} \mathrm{r}} \quad, \mathrm{y}=\mathrm{k}_{\mathrm{s}} \mathrm{r}
$$$$
\frac{\overline{\mathrm{A}}}{\overline{\mathrm{B}}}= \pm \hat{\theta} \frac{\cos \phi}{\sin \phi}-\hat{\phi} \cos \theta \begin{array}{r}
\sin \phi \\
\cos \phi
\end{array}
$$

$$
\frac{\overline{C_{1}}}{\overline{\mathrm{C}}}= \pm \hat{\theta} \cos \theta \frac{\cos \phi}{\sin \phi}-\hat{\phi}\left(-1+2 \cos ^{2} \theta\right) \begin{aligned}
& \sin \phi \\
& \cos \phi
\end{aligned}
$$

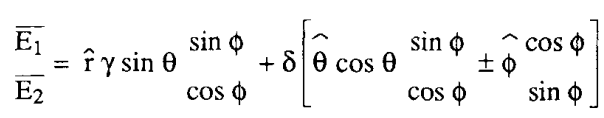

$$
\begin{aligned}
\overline{F_{1}}=\hat{\mathrm{F}} \kappa \sin \theta \cos \theta \begin{array}{l}
\sin \phi \\
\overline{\cos \phi}
\end{array} & \\
& +\zeta\left[\hat{\theta}\left(-1+2 \cos ^{2} \theta\right) \begin{array}{l}
\sin \phi \\
\cos \phi
\end{array} \hat{\phi} \cos \theta \begin{array}{r}
\cos \phi \\
\sin \phi
\end{array}\right]
\end{aligned}
$$

$$
\begin{aligned}
& \alpha=-1-\frac{j}{y} \\
& \beta=3 j\left(1-\frac{3}{y^{2}}+\frac{3 j}{y}\right) \\
& \gamma=-2\left(\frac{1}{y}+\frac{j}{y^{2}}\right) \\
& \delta=-j+\frac{1}{y}+\frac{j}{y^{2}} \\
& \kappa=18 j\left(\frac{1}{y}+\frac{3 j}{y^{2}}-\frac{3}{y^{3}}\right) \\
& \zeta=3\left(-1+\frac{6}{y^{2}}-\frac{3 j}{y}+\frac{6 j}{y^{3}}\right)
\end{aligned}
$$

Substituting (10 - 12) into (3) and (4) yield the final expressions for the scattered fields.

$\overline{E^{s}}=K_{1}\left[\frac{3}{2}\left(a_{1} \alpha \bar{A}-j b_{1} \overline{E_{2}}\right)+\frac{5}{6}\left(j a_{2} \beta \overline{C_{1}}+b_{2} \bar{F}\right)\right]$

$\overline{\mathrm{H}^{s}}=\frac{-\mathrm{K}_{1}}{\eta}\left[\frac{3}{2}\left(b_{1} \alpha \overline{\mathrm{B}}+j \mathrm{a}_{1} \overline{\mathrm{E}_{1}}\right)+\frac{5}{6}\left(j b_{2} \beta \overline{\mathrm{C}}-\mathrm{a}_{2} \overline{\mathrm{F}}\right)\right]$

where

$$
K_{1}=j K_{0} E_{0}
$$

To construct the phase matrix [10], coordinate transformations are performed on (13) to permit arbitrary incident angles and polarizations. The detailed description of the transformation is given by Fung and Eom[11]. The phase matrix associated with the first two Stokes parameters is constructed from the resulted scattered fields as follows: 


$$
\bar{P}=\left[\frac{\left|E_{d}\right|^{2}}{\eta}\right]^{-1} \frac{r^{2}}{2} \operatorname{Re}\left[\begin{array}{cc}
\left(E_{v}^{s} H_{h}^{s *}\right)_{v-i n c} & \left(E_{v}^{s} H_{h}^{s *}\right)_{h-i n c} \\
\left(-E_{h}^{s} H_{v}^{s}\right)_{v-i n c} & \left(-E_{h}^{s} H_{v}^{s}\right)_{h-i n c}
\end{array}\right]
$$

The phase matrix in (14) is related to the volume fraction of the scatterers embedded in the medium due to the range dependent parameters $\alpha, \beta, \gamma, \delta, \kappa$, and $\zeta$. The range $\mathrm{r}$ is related to volume fraction $f$ of spherical scatterers by $r=\left(\frac{v}{f}\right)^{1 / 3}$, where $v=\frac{4}{3} \pi a^{3}$ [12]. The radiative transfer approach [1] is used in calculating the intensity scattering using the phase matrix (14).

\section{RESULTS AND DISCUSSION}

The geometry of scattering from inhomogeneous layer embeded with spherical scatterers is shown in Fig. 2. A good fit is obtained for the sea ice data using the current scatter model with the phase matrix given by (14). The estimated sea ice parameters using the current scattering model are: air bubbles' volume fraction $\mathrm{f}=20 \%$, and average air bubble radius $a=0.56 \mathrm{~mm}$. Fig. 3 . shows the comparison of theoretical results with experimental data collected from multi year Arctic sea ice at $13 \mathrm{GHz}[4]$. The sea ice has a very smooth surface and the thickness is greater than $3 \mathrm{~m}$.

In order to better understand the dense medium scattering process, further studies on the relation between the scatterer volume fraction, $f$, and the range, $r$, are necessary

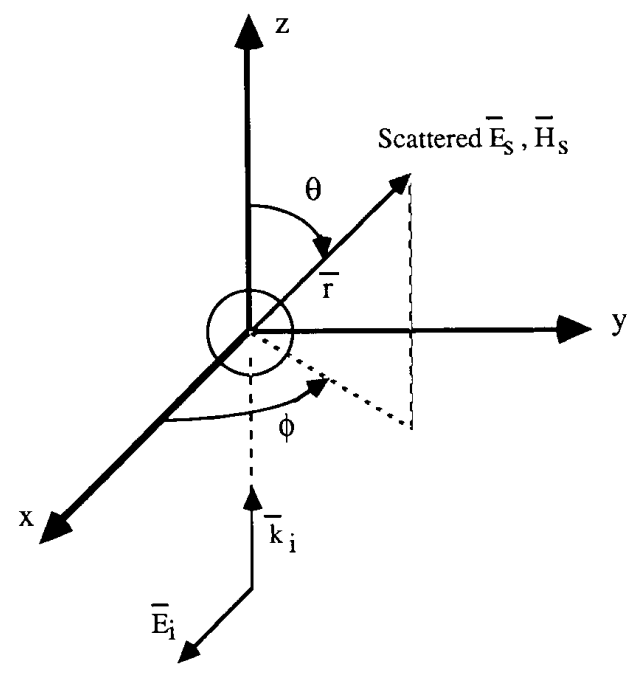

Fig. 1. Geometry of scatter problem.

\section{REFERENCES}

[1] A.K.Fung and H.J.Eom, "Application of combined rough surface and volume scattering theory to sea ice and snow backscatter," IEEE Trans. Geosci. Remote Sensing, vol. GE-20, pp528-536, Oct. 1982.

[2] R.T.Shin and J.A.Kong,"Radiative transfer theory for active remote sensing of a homogeneous layer containing spherical scatterers," J. App. Phys., vol. 52, no. 6, pp 4221-4230, June 1981.

[3] H.C.Hottel, A.F.Sarofim, W.H.Dalzell, I.A.Vasalos, "Optical Properties of coatings, effect of pigment concentration," AIAA J., vol. 9, no. 10, pp. 1895-1898, Oct 1971.

[4] R.G.Onstott, R.K.Moore, and W.F.Weeks, "Surface-based scatterometer results of Artic sea ice," IEEE Trans. Geosci. Electron., vol. GE-17, pp 4712-4717, Nov. 1974.

[5] J.W.Bredow,"A laboratory investigation into microwave backscattering from sea ice," RSL Technical Report 82401, August 1989.

[6] J.A.Stratton, Electromagnetic Theory. McGraw-Hill 1941, pp. 558, 564, 571.

[7] M.A.Karam and A.K.Fung,"Some basic properties of extinction, scattering and absorption efficiencies of a Mie sphere,"

[8] W.J.Wiscombe,"Improved Mie scattering algorithms." Applied Optics, vol. 19, no. 9, May 1980.

[9] G.T.Ruck,"Radar cross section handbook," Plenum Press, vol. 1, 1970, pp. 160-161.

[10] A.Ishimaru, Wave Propagation and Scattering in Random media, vol. 1. Academic Press, 1978, pp. 34-35.

[11] A.K.Fung and H.J.Eom,"A study of backscattering and emission from closely packed inhomogeneous media," IEEE Trans. Geosci. Remote Sensing, vol. GE-23, no. 5, September 1985.

[12]G.H.Goedecke,"Radiative transfer in closely packed media," GOSA, vol.67, no. 10, pp. 1339-1348, Oct. 1977. 


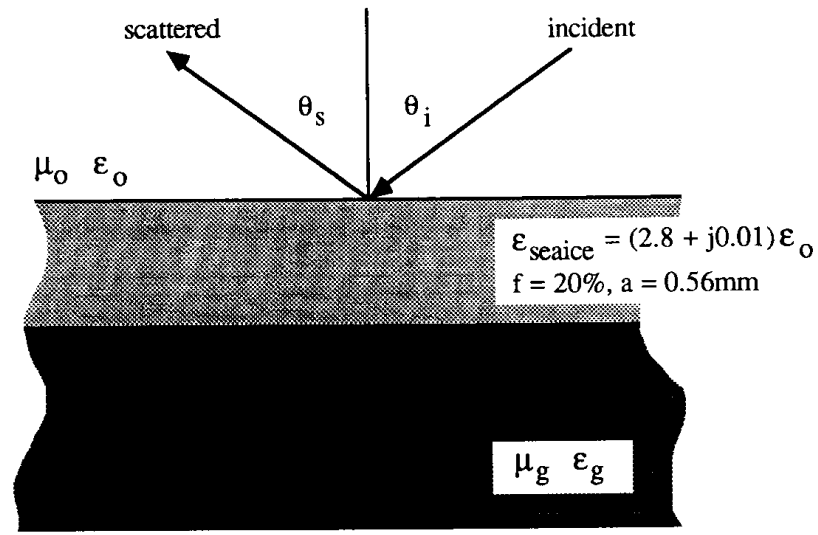

Fig. 2. Scattering geometry of the dense medium layer.

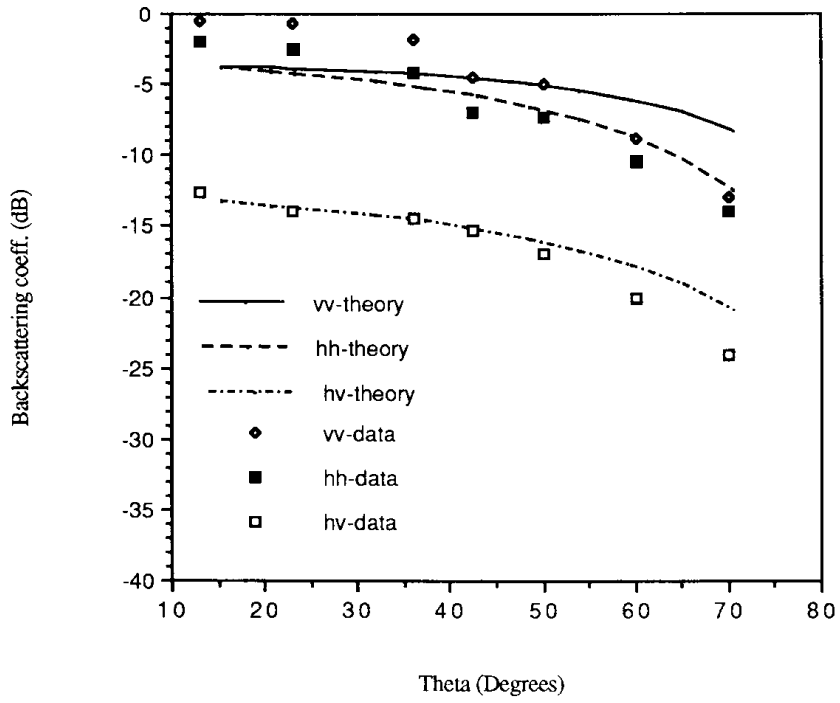

Fig. 3. Comparison of sea ice backscatter data, freq $=13 \mathrm{GHz}$, with theoretical results. 